\title{
Spectral analysis of a transitional separated-reattached flow using Fourier and wavelet transforms
}

\author{
I. E. Abdalla \& M. J. Cook \\ Institute of Energy \& Sustainable Development, De Montfort University, \\ Leicester, $U K$
}

\begin{abstract}
Large-eddy simulations (LES) of transitional separating-reattaching flow over a square surface mounted obstacle (SSMO) and a forward-facing step (FFS) have been performed. The Reynolds number based on the uniform inlet velocity and the obstacle height is $4.5 \times 10^{3}$. A dynamic subgrid-scale model is employed in this work. The mean LES results compare favourably with the available experimental and DNS data.

This paper addresses the characteristic shedding modes associated with the separated-reattached flows on the SSMO and the FFS and sheds light on the use of the wavelet transform (WT) in extracting the content of a time history of a (velocity and/or pressure) signal compared to the traditional Fourier transform (FT). The turbulence spectra for the geometries revealed amplified frequency modes both upstream and downstream of the separation edge with those associated with the SSMO showing more clearly compared to the FFS. A frequency peak was detected at a location upstream of the separation line and immediately above the SSMO. The value of this frequency suggests that the upstream separated region is unstable via the Kelvin-Helmholtz instability and the peak can not be attributed to the flapping of the separated shear layer which is a phenomenon commonly associated with this class of flows. The WT captured events that are characterised by narrow periods (scales) and which happened over shorter times. Such events are smoothed out by the Fourier transform indicating the superiority of the WT over the FT. Keywords: large-eddy simulation, wavelet and Fourier transform, spectral analysis, transitional to turbulence.
\end{abstract}




\section{Introduction}

Turbulent and transitional flows over obstruction such as the SSMO and the FFS are an important group of separated-reattached flows that occur in many industrial and environmental applications. For example, control of boundary layers, river flows, wind loads on structures, and the spread of pollutants in the vicinity of buildings.

Separated-reattached flows over an obstruction is quite complicated when compared with other bluff body geometries such as the backward-facing step and the blunt leading edge plate aligned to a flow field. The complication comes as a result of an additional separation in the upstream region displayed by the obstruction leading to a closed or open bubble (Satür et al [1]). For the SSMO, there exist few studies which are focused on the very basic features of this class of flows such as the variation of the mean reattachment length with the Reynolds number (Tropea and Gackstatter [2]), the influence of the obstacle aspect ratio $\left(\frac{l}{h}\right)$ on the mean reattachment length (Bergeles and Athanassiadis [3]), the effect of varying the blockage ratio (Durst and Rastogi [4]).

Little work has been published on either laminar or turbulent separated flows over the FFS flow. Recently, Satür et al [1] and Wilhelm et al [5] performed experimental and computational work respectively to study the laminar separation on a forward facing step for Reynolds numbers as low as 30 . This work is mainly focused on the flow structure and the instability causing three-dimensionality of the flow downstream of the leading edge.

Previous studies for separated-reattached flows have identified specific frequency modes associated with some physical phenomena of the separatedreattached flows, such as the shedding frequency, in addition to a lower one which is attributed to flapping of the shear layer (Kiya and Sasaki [6]). The main technique used by the researchers to identify these range of frequencies is through FT to a time series of the velocity and pressure field with the aid of flow visualization. Kaiser [7] suggests that the FT method is inaccurate and inefficient for time-frequency localisation. The spectra of transitional/turbulent flow usually contain a range of frequencies. For analysis where a predetermined scaling may not be appropriate because of a wide range of dominant frequencies (such as signals resulting from a transitional/turbulent flows), a method of timefrequency localization that is scale dependent, such as wavelet analysis, might be more successful.

The aim of this paper is to shed light on the use of the WT as a method of extracting the content of a time series of the velocity and pressure fields obtained from a large-eddy simulation on separated-reattached flow on a SSMO and a FFS and discuss the processes responsible for the content of the spectra (shedding and interaction of the coherent structures). The WT method is not commonly used in this field and its strengths/weaknesses are not well known when applied to data from unsteady turbulent flows. The objectives are: (i) to identify the amplified 
frequencies associated with the SSMO and FFS, and (ii) to compare the spectra details using the FT and WT and highlight the strengths and weaknesses of the two methods.

\section{Details of numerical computation}

The governing equations are discretised on a staggered grid using the finite volume method. Any small-scale (smaller than the control volume) motions are averaged out and have to be accounted for by a subgrid-scale model. A standard dynamic subgrid-scale model in cartesian co-ordinates has been employed in the present study. The ratio $\nu_{s} / \nu$ is zero in the laminar region before transition occurs and starts to increase shortly after the separation line reaching a maximum value of about 9 around the mean reattachment location and dropping down to about 5 after reattachment. The explicit second order AdamsBashforth scheme is used for the momentum advancement. The Poisson equation for pressure is solved using an efficient hybrid Fourier multigrid method. The spatial discretisation is secondorder central differencing which is widely used in LES owing to its non-dissipative and conservative properties. More details of the mathematical formulation and numerical methods have been reported elsewhere by Yang and Voke [8].

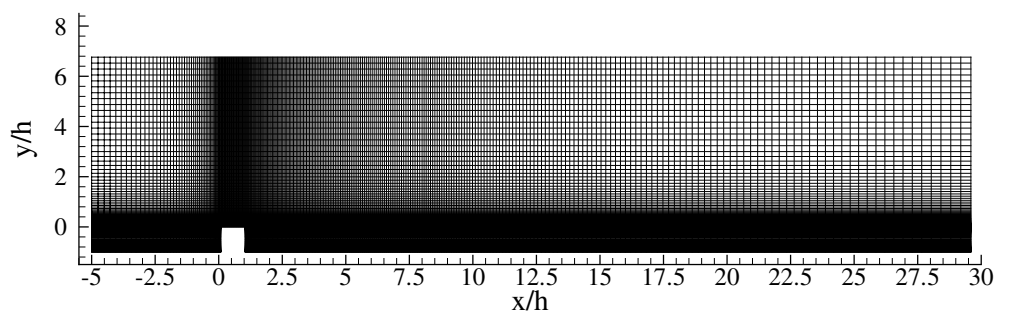

Figure 1: The computational domain and mesh used for the first simulation of the SSMO.

Two simulations were performed for the obstacle case. In the first simulation (figure 1) $288 \times 128 \times 64$ cells along the streamwise, wall-normal and spanwise directions respectively were employed. The blockage ratio is 8 and the spanwise dimension of the domain is $4 h$. A free-slip but impermeable boundary is applied at the lateral boundary, periodic boundary along the spanwise and no-slip boundary conditions are used at all other walls. At the inflow boundary, a uniform velocity profile is applied and the Reynolds number based on the inflow velocity and obstacle height is 4500 . At the outflow boundary, a convective boundary condition is applied. In terms of wall units based on the friction velocity downstream of reattachment at $x / h=27$, the streamwise mesh sizes vary from $\Delta x^{+}=6.77$ to $\Delta x^{+}=43.04$, while $\Delta z^{+}=10.625$ and at the wall $\Delta y^{+}=1.28$. The time step used in this simulation is $4.75 \times 10^{-6}$ second $\left(0.001425 \frac{h}{U_{0}}\right)$. The simulation ran for 129,000 time steps, equivalent to more than 5 flow passes through the 
domain (or residence times) to allow the transition and turbulent boundary layer to be established, i.e. the flow to have reached a statistically stationary state. The averaged results were gathered over a further 249,900 time steps, with a sample taken every 10 time steps (24,990 samples) averaged over the spanwise direction, corresponding to more than 10 flow passes or residence times.

The second simulation used $320 \times 220 \times 64$ cells for $0.25 \mathrm{~m} \times 0.15 \mathrm{~m} \times 0.04 \mathrm{~m}$ resulting in a blockage ratio of 15 . The streamwise mesh sizes vary from $\Delta x^{+}=$ 6.09 to $\Delta x^{+}=19.988$, while $\Delta z^{+}=10.96$ and at the wall $\Delta y^{+}=1.14$. The averaged results gathered from this simulation show no significant changes in the mean reattachment length from the first simulation. The same computational domain used in the second SSMO simulation was adopted for the FFS (with the step leading edge again located at $\mathrm{x} / \mathrm{h}=5$ ). The FFS case ran for a total of 404,000 time steps with the sampling for the mean field started 100,000 after the start of the run.

\section{Mean flow field}

An important parameter characterising a separated-reattached flow is the time mean position of the reattachment. The mean streamlines and the mean axial velocity profile at the first cell from the solid boundary (a method used to determine the mean reattachment location) are shown in figure 2(a) and $b$ respectively and indicate that the mean reattachment length is $\approx 15.5 \mathrm{~h}$. The predicted mean reattachment length compares well with the experimental measurements of $15.5 \mathrm{~h}$ reported by Tropea and Gackstatter [2] which is the benchmark used in this work for the current LES of the SSMO. Other values include Bergeles and Athanassiadis [3] $\left(x_{R} / h=11\right)$, Durst and Rastogi [4] $\left(x_{R} / h=16\right)$, all of which are under a turbulent condition with high free-stream turbulence. Similar scatter was reported for the fence geometry including Tropea and Gackstatter [2] $\left(x_{R} / h=17\right)$, the DNS study of Orellano and Wengle [9] $\left(x_{R} / h=13.2\right)$ and the experiment of Larsen [10] $\left(x_{R} / h=11.7\right)$. Overall, the LES simulation has predicted the line mean position of the reattachment well.

Similarly, figures 3(a), and (b) are the corresponding mean stream line and the mean streamline velocity profiles at the first cell from the solid surface for the FFS flow. The predicted mean reattachment length downstream the separation line read from the two figures is $8.1 \mathrm{~h}$. Experimental and computational studies for the FFS are few and those which exist have predicted a shorter length than $8.1 \mathrm{~h}$. Ko [11] simulation predicted this length as $5.5 \mathrm{~h}$ and the measured value from Moss and Baker [12] experiment is 4.8h. Similarly, the work of Bergeles and Athanassiadis [3] showed that the mean reattachment length downstream an extended obstacle is of order $4 \mathrm{~h}$. In contrast with the current simulation it appears that the LES has over-predicted this parameter. But once again the difference is thought to be due to the high Reynolds number and the nature of turbulent flows in the work cited here. For laminar separation, as in the current case, the transition could be delayed leading to a longer bubble than the case for turbulent separation. A relevant type of flow to the FFS is the blunt plate experiment of Castro and 
(a)

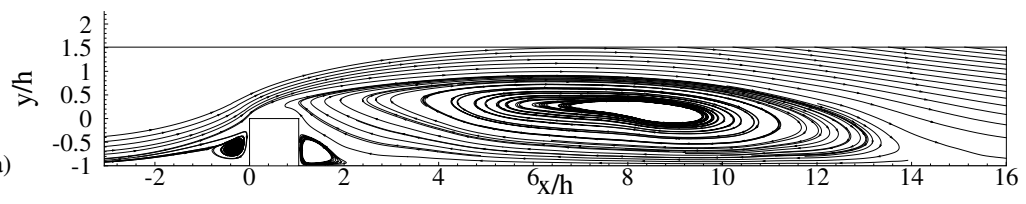

(b)

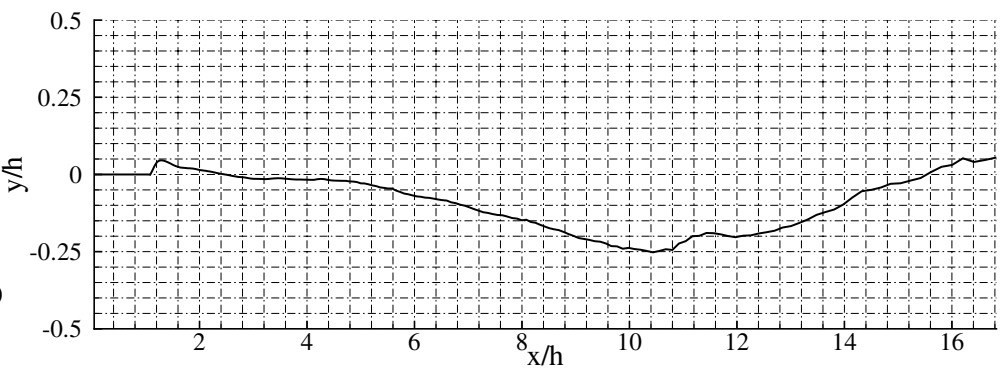

Figure 2: LES prediction of flow over the SSMO: (a) Mean streamlines, (b) profile of mean axial velocity at the first cell from the solid surface along the streamwise direction.

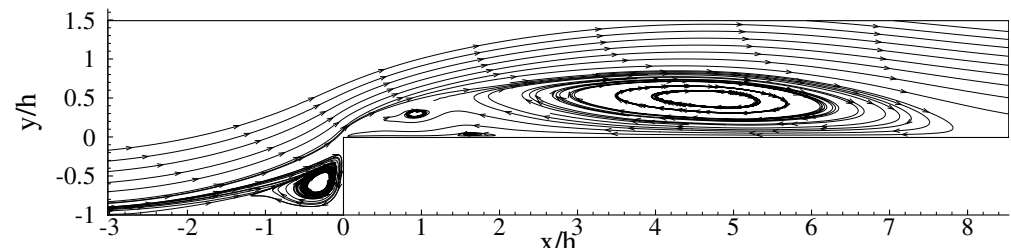

(a)

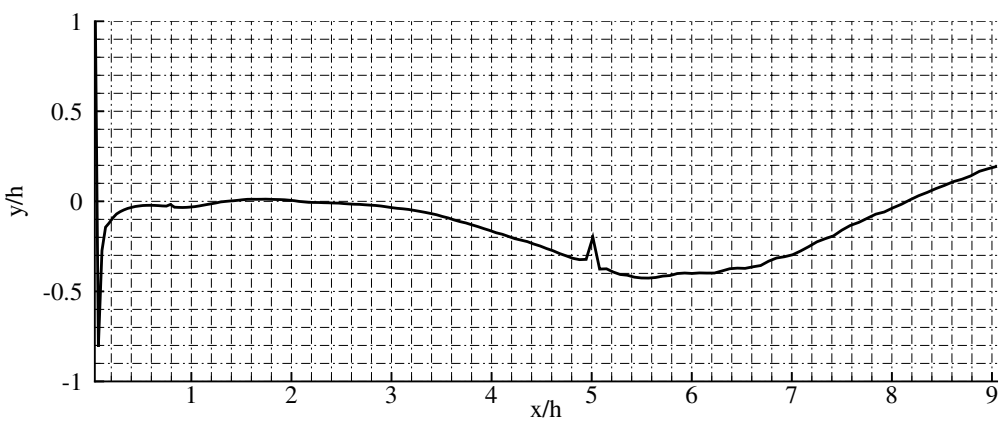

(b)

Figure 3: LES prediction of flow over the FFS: (a) Mean streamlines, (b) profile of mean axial velocity at the first cell from the solid surface along the streamwise direction.

Epik [13] $\left(R e_{D}=6500\right)$ in which the reattachment is reported to be 7.7D, where $D$ is the plate thickness. This is comparable to the mean reattachment length for the current simulation. 


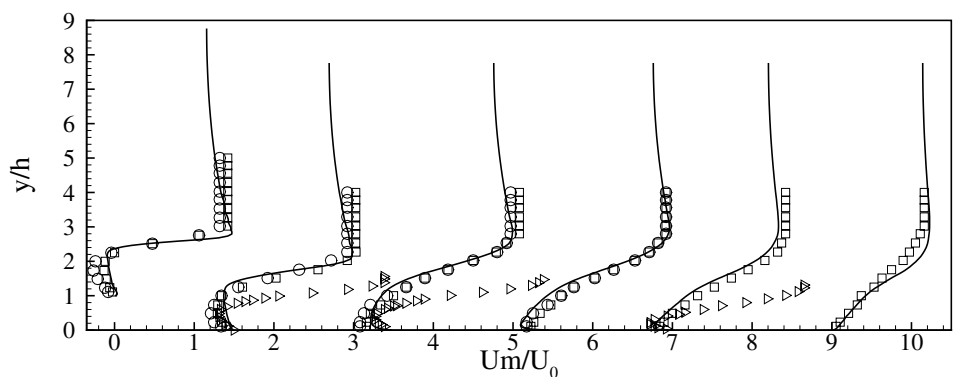

Figure 4: The SSMO flow: profiles of mean streamwise velocity $U_{m} / U_{0}$ at six streamwise locations measured from the separation line (leading edge). Left to right $x / x_{R}=0.05,0.2,0.4,0.6,0.8,1.025$. Also shown are measurements by Tropea and Gackstatter [2] (triangle), Larsen [10] (square) and the DNS data of Orellano and Wengle [9] (circle symbol) at $\mathrm{Re}=3,000$.

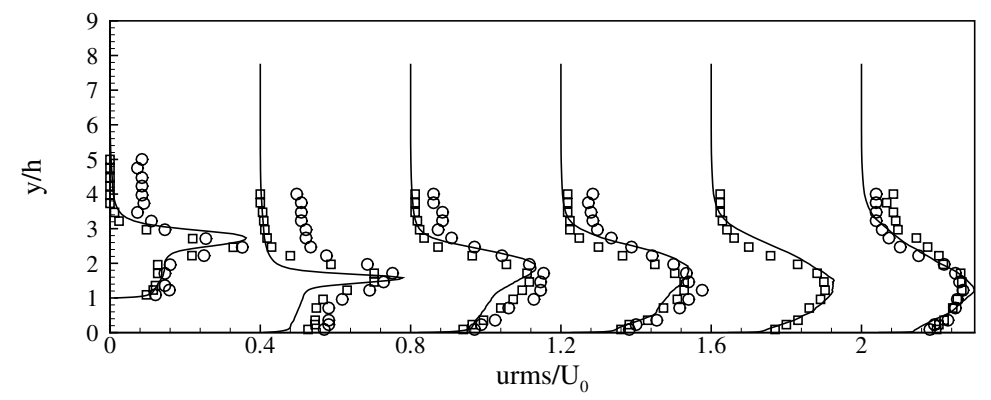

Figure 5: The SSMO flow: profiles of mean streamwise turbulent intensity $u_{r m s} / U_{0}$ at six streamwise locations measured from the separation line (leading edge). Left to right $x / x_{R}=0.05,0.2,0.4,0.6,0.8,1.025$. Also shown are measurements by Larsen [10] (square) and the DNS data of Orellano and Wengle [9] (circle) at $\mathrm{Re}=3,000$.

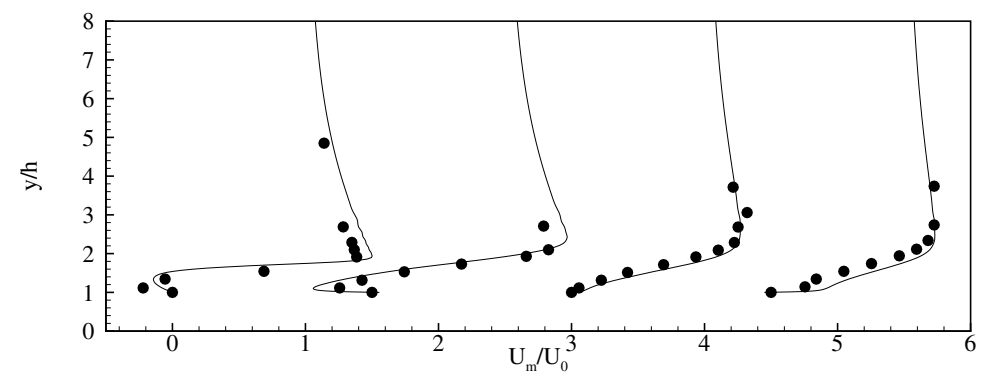

Figure 6: The FFS flow: profiles of mean streamwise velocity $U_{m} / U_{0}$ at four streamwise locations measured from the separation line (leading edge). Left to right $x / x_{R}=0.208,0.625,1.04$, and 1.25 . Also shown are measurements by Moss and Baker [12] (circle) at Re=46,000. 
Figure 4 compares the mean streamwise velocity distribution $\bar{U} / U_{0}$ at 6 locations downstream of the separation line with the experimental data of Tropea and Gackstatter [2] (available only at 3 locations), Larsen [10] and the DNS data of Orellano and Wengle [9]. The results show good agreement with the data of Larsen [10] and the DNS data of Orellano and Wengle [9]. The freestream velocities of the data from Tropea and Gackstatter [2] are bigger than those predicted by the LES and the other two results, and peak at lower y-values. This is attributed to the difference in blockage ratio used by Tropea and Gackstatter [2] which is very low $(=2), 5$ in the case of Orellano and Wengle [9] and 8 for the current LES.

Profiles of the rms streamwise velocity, $u_{r m s}$, normalised by $U_{0}$, at the same six stations are shown in figure 5. The agreement between the LES results and the data of Larsen [10] and the DNS data of Orellano and Wengle [9] is encouraging. No measured data were presented by Tropea and Gackstatter [2].

Data for the mean velocity profiles for the FFS flow at low Reynolds number does not exist. Therefore, the results were compared with experimental data by Moss and Baker [12] at higher Reynolds number $\left(R e_{h}=46000\right)$. This is thought not to be detrimental to the comparison and any discrepancy will be discussed taking this fact into consideration. Figure 6 compares the mean streamwise velocity distribution $\bar{U} / U_{0}$ at 4 locations downstream of the separation line with the experimental data of Baker [12]. The agreement between the experimental and LES results is reasonably good. At the location $x / x_{R}=0.625$ the peak negative value is slightly higher than the experimental value. This could be due to the low Reynolds number for the LES case leading to a stronger back flow and low mixing at this specific region of the recirculation region downstream the step. The profiles of the rms streamwise velocity, $u_{r m s}$, normalised by $U_{0}$ (not shown here), at the same four locations presented in figure 6 also display good agreement when compared with the data of Moss and Baker [12].

\section{Spectra using Fourier and wavelet transforms}

For the current work, a well tested code that uses the FT methods for autocorrelation is used to process the data. For the WT, the Morlet wavelet is used and a code developed by Torrence and Compo ([14]) was modified to perform the analysis for the time series signals shown in this section. The WT analysis produces a $2 \mathrm{D}$ picture showing wavelet power concentration in time (x-axis) vs scales or period (y-axis) (which is an approximate measure to the Fourier period of the signal). For more details on wavelet transform techniques, the reader is advised to consult Torrence and Compo [14]. A total of 24990 samples at each point taken every 10 time-steps with time step $=4.75 \times 10^{-6}$ seconds (sampling frequency $21.053 \mathrm{kHz}$ ) were collected for the SSMO case. This corresponds to a total period of 1.187025 seconds. For the FFS simulation, 32,312 samples were collected with each sample taken every 10 time-steps with time step $=1.5 \times 10^{-6}$ seconds (sampling frequency $66.67 \mathrm{kHz}$ ). This corresponds to a total period of 0.484680 seconds. 
It is essential to choose a set of scaling parameters $s$, such that the wavelet transform adequately samples all the frequencies present in the time series. The smallest resolvable scale, $s_{0}$, is usually selected as a multiple of the time resolution $d t$ and for the current studies is chosen as $2 d t$ for the SSMO and FFS data. Torrence and Compo [14] recommended that the largest scale chosen should be less than 1/2 the length of the entire time series. For the two cases presented here, the largest scale chosen is of order one third of the total time span and hence, no interest in event with long periods is sought. However, larger periods are also investigated to shed light on the difference of choosing this parameter associated with WT.

Shown in figures 7(a) and (c) are the time history for the velocity $u$ and $v$ at the position described by the co-ordinates $(\mathrm{h}=-0.375, \mathrm{y} / \mathrm{h}=0.04, \mathrm{z} / \mathrm{h}=2)$, immediately upstream and slightly above the leading edge of the SSMO. The WT spectra based, on scales of order one third of the total sampling time, is shown in figures 7(b) and (d) and the spectra based on the FT for these velocities appear in figure 7(e). The FT spectra clearly show a sharp frequency peak (band) centered at approximately $105 \mathrm{~Hz}$ for both velocity components (the normalised value is $5.425 \frac{U_{0}}{x_{R}}$ ). The wavelet spectra for the velocity $\mathrm{U}$ (figure 7(c)) reveal power concentration associated with four instances of time. The major power is centered around $t \approx 0.26 \mathrm{~s}$ with a band scale $\leq 0.2$. Linked to this event, there is another event which is characterised by less power compared the major event, having a lower band $(0.1 \leq$ scale $\leq 0.2)$ and centered around $t \approx 0.5$ s (the two events are thought to be associated with the peak noticed in the FT spectra). The wavelet spectra also indicates that an event with lower power concentration and much narrower band $(0.09 \leq$ scale $\leq 0.11)$ has taken place between $0.85 \leq t \leq 1.2$. Also, towards the end of the sampling period, the WT spectra indicates that the major event (or velocity peak) could possibly reoccur. The last point is clear from the WT spectra for the velocity $v$ (figure 7(d)) which shows power concentration shortly after the start of the sampling and towards the end of the time history.

Comparing the events shown by the WT spectra with the time series, it is clear that the WT is able to interpret exactly the content of the event displayed in the history for each velocity component. Whenever the signal shows a sharp positive or negative peak which is most likely an indication of an event (shedding, or pairing of large-scale structures), it was captured in the WT spectra with its representative magnitude and at the exact time where the process happened. All these features represent the benefits of using WT to perform spectral analysis for unsteady turbulent flow. One of the critical features of the WT spectra is the fact that it provides only qualitative results (many criticise the method for this feature). However, it does give a clear picture of the extent of the event (amplitude) and any smaller events associated with it and the time of occurrence and possibly the cyclic behaviour of the major event. This information could be useful in controlling such events (damping or exciting as required for specific applications)

It is essential to set an appropriate range of scales for the WT spectra to be realistic. As an example, the largest scale for the velocity $u$ in figure 7(b) has been increased in figure 7(f) in order to seek events with higher period. It is clear that the content shown by this figure is a little misleading when compared with the 
corresponding time history of the signal where events with lower concentration and those occurring at shorter times have vanished. This confirms that care should be taken when using WT spectra for turbulent flow data analysis.

For the FFS flow and for a point similar to the location of the SSMO discussed above $(\mathrm{x} / \mathrm{h}=-0.225, \mathrm{y} / \mathrm{h}=0.2, \mathrm{z} / \mathrm{h}=2)$, the time series for the velocities $u$ and $w$ are shown in figures $7(\mathrm{~g})$ and (i) respectively with the WT spectra shown in figures 7(h) and (j) respectively. The FT spectra is displayed in figure 7(k). The interesting point here is the fact that the FT spectra does not show any particularly amplified frequency. However, the WT spectra for the velocity $u$ shows power concentrations both at the beginning of the sampling period and towards the end while that for the velocity $\mathrm{w}$ shows a clear and significant concentration shortly after the start of the sampling process. The reason for these events not being shown in the FT spectra is probably due to the short total time $(\approx 0.45 \mathrm{sec}$ compared to $1.25 \mathrm{sec}$ for the SSMO) for the samples collected in the FFS which may be too short to resolve this particular frequency. However, the WT spectra shows that there are significant events at the location mentioned above for the SSMO flow. It is worth pointing out that this detected frequency is most likely due to Kelvin-Helmholtz (K-H) instability rather than being attributed to the flapping of the shear layer since its reduced value is much higher than the later which is of order $0.15 \frac{U_{0}}{x_{R}}$.

For the SSMO and downstream the separation line ( $\mathrm{x} / \mathrm{h}=1.65, \mathrm{y} / \mathrm{h}=0.85, \mathrm{z} / \mathrm{h}=2)$, figures $7(\mathrm{l})$ shows a fading frequency peak (centered at $\mathrm{f}=200 \mathrm{~Hz}$ ) which most likely represents vortex shedding/pairing of vortices rolling-up at the fundamental instability frequency of the separating shear layer. A similar value is reported for the FFS downstream the leading edge (not shown here).

\section{Conclusion}

Analysis of the velocities and pressure fields signals predicted by LES for a transitional separated-reattached flow over a SSMO and FFS was performed employing the FT and the WT. The turbulence spectra for the geometries revealed amplified frequency modes both upstream and downstream of the separation edge with those associated with the SSMO more apparent compared to the FFS. The value of amplified frequency upstream strongly suggest that the upstream separated region is unstable via the $\mathrm{K}-\mathrm{H}$ instability and the frequency could not be attributed to the flapping of the shear layer as commonly reported for such class of flows. Those which appear downstream the separation line are attributed to the shedding and pairing activities of large-scale motions dominating the separated boundary layer.

The WT correctly interpreted the content of the time signal for the presented data. Whenever the time signal indicates the occurrence of a certain event, it was marked in the WT spectra with its representative amplitude. The WT was also able to capture events that are characterised by narrow periods (scales) and which happened over shorter times. Such events are smoothed out by the Fourier transform indicating the superiority of the WT over the FT in revealing a signal 

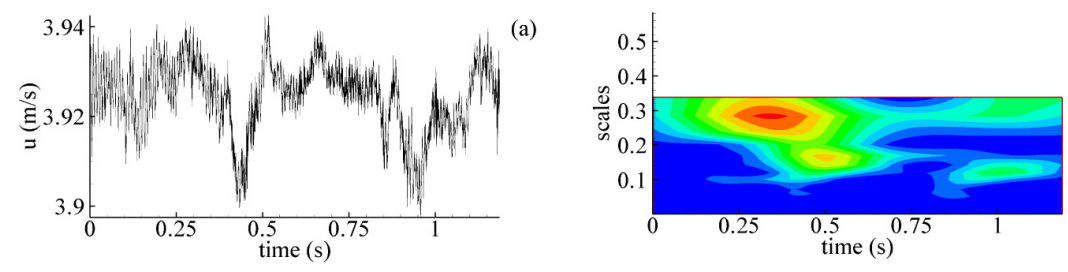

(b)
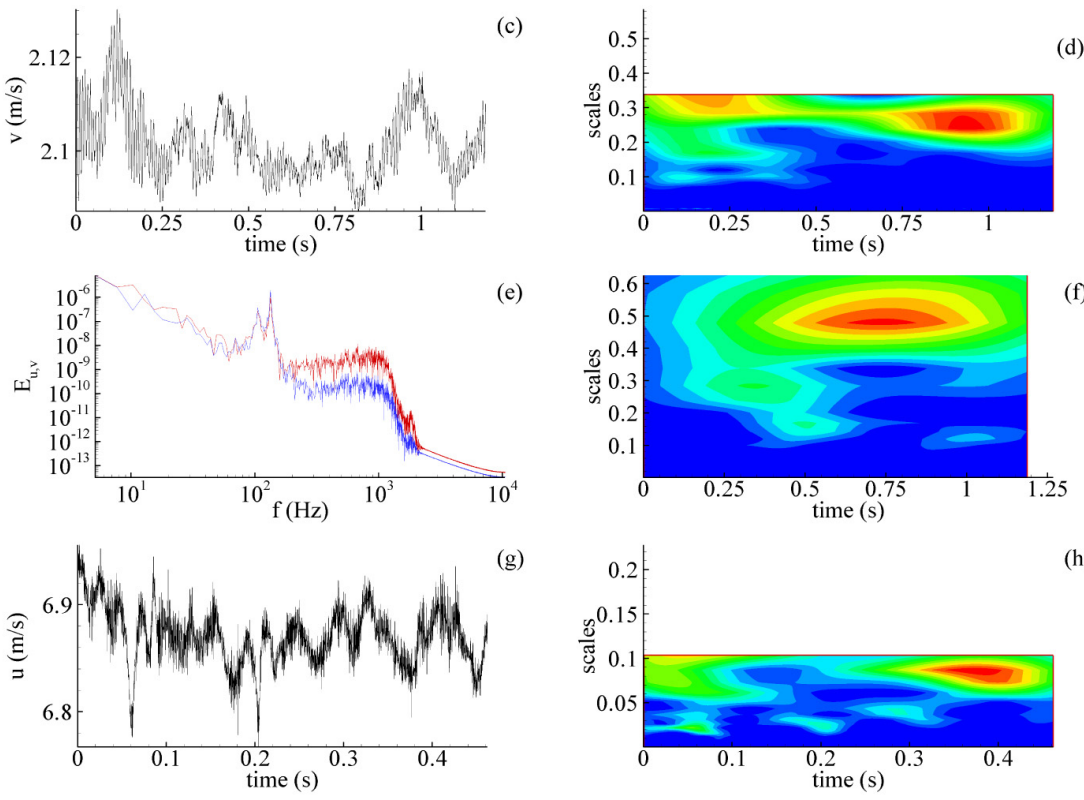

(h)
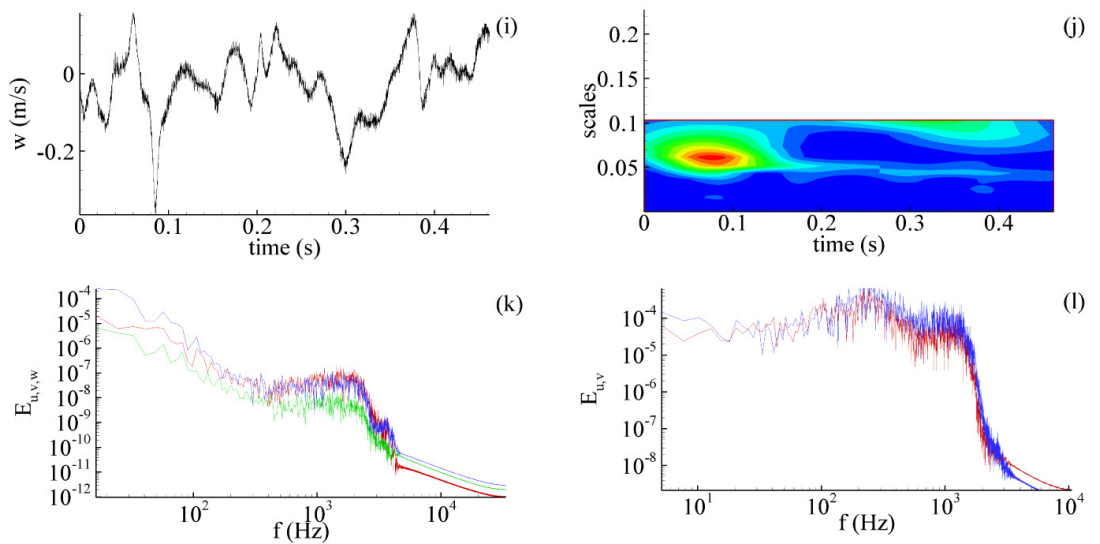

Figure 7: Time signal, FT and WT spectra for the SSMO (a), (b), (c), (d), (e), (f) and (l) and FFS (g), (h), (i), and (j). 
contents. However, it was found that appropriate range of scales must be selected for the WT in order to adequately sample the existing frequencies within a signal and avoid misleading interpretation.

\section{References}

[1] Satür, H., Gyr, A. and Kinzelbach, W. Laminar separation on a forward facing step. Eur. J. Mechj. B/Fluids 18, 675-692, 1999.

[2] Tropea CD, Gackstatter R. The flow over two-dimensional surface-mounted obstacles at low Reynolds number, Journal of Fluids Engineering; 107:489494, 1984.

[3] Bergeles G, and Athanassiadis N. The flow pass a surface mounted obstacle. ASME Journal of Fluid Engineering; 105:461-463, 1983.

[4] Durst F, Rastogi AK. Turbulent flow over two-dimensional fences, in Turb. Shear Flows 2. Springer Verlag: Berlin, 218-231, 1980.

[5] Wilhelm, D., Härtel, C., and Kleiser, L. Computational analysis of the two-dimensional-three-dimensional transition in forward-facing step flow. $J$. Fluid Mech; 179:439-468, 1987.

[6] Kiya M, Sasaki K. Structure of a turbulent separation bubble, J. Fluid Mech; 137:83-113, 1983.

[7] Kaiser G. A Friendly Guide to Wavelets. Cambridge, MA: Birkhuser, 300pp, 1994.

[8] Yang, Z. and Voke, R.P. Large-eddy simulation of separated leading-edge flow in general co-ordinates. International J. Numer. Meth. Engng. 49, pp. 681696, 2000.

[9] Orellano A, Wengle H. Numerical simulation (DNS and LES) of manipulated turbulent boundary layer flow over a surface-mounted fence. Er. J. B - Fluids, 19:765-788, 2000.

[10] Larsen, P. S. Database on tc-2C and tc-2D fence-on-wall and obstacle-onwall test case. Report AFM-ETMA 95-01, ISSN 0590-8809; TU Denmark, 1995.

[11] Ko, S. H. Computation of turbulent flows over backward and forward-facing steps using a near-wall Reynolds stress model. CTR Annual Research Briefs, Stanford University/NASA Ames, 75-90, 1993.

[12] Moss W. D., Baker S. Re-circulating Flows Associated with Twodimensional Steps, Aero Quart., 151-172, 1980.

[13] Castro IP, Epik E. Boundary layer development after a separated region. Journal of Fluid Mechanics, Vol.374(), pp.91-116, 1998.

[14] Torrence C, Compo GP. A practical guide to wavelet analysis. Bulletin of the American Meteorological Society, 61-77, 1998. 